\title{
Benchmark calculation of $p-{ }^{3} \mathrm{H}$ and $n-{ }^{3} \mathrm{He}$ scattering
}

\author{
M. Viviani, ${ }^{1}$ A. Deltuva, ${ }^{2}$ R. Lazauskas,${ }^{3}$ A. C. Fonseca, ${ }^{4}$ A. Kievsky, ${ }^{1}$ and L. E. Marcucci ${ }^{1,5}$ \\ ${ }^{1}$ INFN-Pisa, 56127 Pisa, Italy \\ ${ }^{2}$ Institute of Theoretical Physics and Astronomy, Vilnius University, LT-10222 Vilnius, Lithuania \\ ${ }^{3}$ IPHC, IN2P3-CNRS/Université Louis Pasteur BP 28, F-67037 Strasbourg Cedex 2, France \\ ${ }^{4}$ Centro de Física Nuclear da Universidade de Lisboa, P-1649-003 Lisboa, Portugal \\ ${ }^{5}$ Department of Physics, University of Pisa, 56127 Pisa, Italy
}

(Received 28 October 2016; published 27 March 2017)

\begin{abstract}
$p-{ }^{3} \mathrm{H}$ and $n-{ }^{3} \mathrm{He}$ scattering in the energy range above the $n-{ }^{3} \mathrm{He}$ but below the $d-d$ thresholds is studied by solving the four-nucleon problem with a realistic nucleon-nucleon interaction. Three different methods-Alt, Grassberger, and Sandhas, hyperspherical harmonics, and Faddeev-Yakubovsky-have been employed and their results for both elastic and charge-exchange processes are compared. We observe a good agreement between the three different methods, thus the obtained results may serve as a benchmark. A comparison with the available experimental data is also reported and discussed.
\end{abstract}

DOI: 10.1103/PhysRevC.95.034003

\section{INTRODUCTION}

Modern studies of nuclear structure and dynamics are mostly based on ab initio calculations using realistic potentials. Due to the complexity of the problem, it is clearly important to have benchmarks between different groups and different techniques in order to test the validity of the existing codes, as well as to establish the numerical accuracy of the solutions. On its turn this may allow a meaningful comparison with experimental data and then serve as a probe of our current understanding of nuclear dynamics. In particular, this program is well suited to be pursued in few-nucleon systems $(A \leqslant 6)$, where several well-controlled numerical techniques have been developed.

The interest in $a b$ initio calculations has been renewed in recent years, i.e., after the advent of the theoretical framework of chiral effective field theory ( $\chi$ EFT), nowadays widely used to derive nuclear potentials and electroweak currents from the symmetries of QCD-the exact Lorentz, parity, and timereversal symmetries, and the approximate chiral symmetry (see, for example, Refs. [1-4]). The test of these new potentials in few-nucleon scattering, where accurate measurements of several observables exist, will give very stringent and critical information.

The three-nucleon system is thoroughly studied and for this case some very accurate benchmarks [5,6] exist. After this achievement, focus has been set on the four-nucleon $(4 N)$ sector. In first place, this system may serve as an ideal "theoretical laboratory" to test our knowledge of the nucleon-nucleon $(N N)$ and three-nucleon $(3 N)$ interactions. In particular, the effects of the $N N P$ waves and of the $3 N$ force are larger than in the $A=2$ or 3 systems, and it is the simplest system where the $3 N$ interaction in channels of total isospin $T=3 / 2$ can be studied. In the second place, there is a number of reactions involving four nucleons which are of extreme importance for astrophysics, energy production, and studies of fundamental symmetries. As an example, the reactions $n+{ }^{3} \mathrm{He}$ and $d+d$ play a key role in the theory of big-bang nucleosynthesis.
Moreover, the potentials derived from $\chi$ EFT contain several unknown parameters - the so-called low energy constants (LECs) - which have to be fixed by comparison with experimental data. This fitting procedure is usually brought forth in $A=2$ and 3 systems, where accurate calculations can be performed since many years and abundant precise experimental data exist. It is therefore of great interest to test the validity of these $\chi$ EFT potentials on independent data, which has not been included in the parametrization. The $4 N$ system, containing several resonances which are not straightforwardly correlated with the $N N$ and $3 N$ sector, presents an ideal test ground.

Nowadays, the $4 N$ bound-state problem can be numerically solved with good accuracy. For example, in Ref. [7] the binding energies and other properties of the $\alpha$ particle were studied by using the $\mathrm{AV}^{\prime}$ [8] $N N$ interaction; several different techniques produced results in very close agreement with each other (at the level of less than 1\%). More recently, the same agreement has also been obtained by considering different realistic $N N+3 N$ force models [9-12].

In recent years, there has also been a rapid advance in solving the $4 N$ scattering problem with realistic Hamiltonians. Accurate calculations of four-body scattering observables have been achieved in the framework of the Alt-GrassbergerSandhas (AGS) equations [13], solved in momentum space [14-16], where the long-range Coulomb interaction is treated by using the screening and renormalization method [17,18]. Also solutions of the Faddeev-Yakubovsky (FY) equations in configuration space [19-23] and the application of the hyperspherical harmonics $(\mathrm{HH})$ expansion method [24] to the solution of this problem have been reported [25,26]. In addition to these methods, the solution of the $4 N$ scattering problem has been obtained also by using techniques based on the resonating group model [27-30]. For applications to $A>4$ systems, see Ref. [31] and references therein.

In a previous work, we presented a benchmark calculation for low-energy $n-{ }^{3} \mathrm{H}$ and $p-{ }^{3} \mathrm{He}$ elastic observables by using the aforementioned AGS, FY, and $\mathrm{HH}$ techniques, and by employing different $N N$ interactions [32]. Nice agreement 
between the results of the three different calculations has been reported; only minor differences were observed for some small polarization observables. It has been concluded that the $n-{ }^{3} \mathrm{H}$ and $p-{ }^{3} \mathrm{He}$ elastic-scattering problem can nowadays be solved with good accuracy.

In the present paper, we extend the benchmark to $p-{ }^{3} \mathrm{H}$ and $n-{ }^{3} \mathrm{He}$ scattering for energies where both channels are open but below the $d$ - $d$ threshold. These calculations present new challenges and are rather complex since the two reaction channels are coupled and involve both total isospin $T=0$ and $T=1$ states. So far, only a few accurate calculations have been performed for these processes [16,23]. Only recently, the AGS method has been extended to the energy regime well above the breakup threshold where the calculations become even more complicated due to nontrivial boundary conditions or singularities [33]. Therefore, we consider the present benchmark as an important step in establishing our current capability to solve the $A=4$ scattering problem. Moreover, our aim is also to provide a set of solid converged results which could represent useful benchmarks for future applications in $A=4$ scattering. The potential used in this paper is the N3LO500 model by Entem and Machleidt [34], based on the $\chi$ EFT approach and derived up to next-to-next-to-next-to-leading order in chiral perturbation theory.

In addition to the desire of improving theoretical tools, it is important to note that, for these reactions, there exist a large amount of experimental data, accumulated during the last 50 years. In particular, below the $d$ - $d$ threshold there exist (since many years) several measurements of the $p-{ }^{3} \mathrm{H}$ elastic differential cross section [35-41], $n-{ }^{3} \mathrm{He}$ elastic differential cross section $[42,43]$ and total cross section [42-45], and $n-{ }^{3} \mathrm{He}$ elastic analyzing powers [46-50]. Regarding the $n-{ }^{3} \mathrm{He} \rightarrow$ $p-{ }^{3} \mathrm{H}$ charge-exchange reaction, there exist mainly measurements of the total cross section $[43,45,51-57]$. For the $p-{ }^{3} \mathrm{H} \rightarrow$ $n-{ }^{3} \mathrm{He}$ charge-exchange reactions, there exist measurements of the differential cross section [58-61] and polarization observables [62-66]. Therefore, another motivation of the present work is to compare theoretical predictions with these data. However, it is important to note that most of these measurements are old, not very accurate, of limited angular ranges, and restricted mainly to unpolarized cross-section data. In fact, only a few experiments have been performed recently $[50,65,66]$ and have an accuracy comparable, for example, to that available for $p$ - $d$ scattering. Therefore, it would be rather useful to have new accurate measurements, in particular of polarization observables. We also remark that previous theoretical studies of these reactions have found a rather high sensitivity to different types of nuclear interaction [16].

The paper is organized as follows: In Sec. II, a brief description of the methods used for this benchmark is reported. In Sec. III, a comparison between the results obtained within the different schemes is shown and the theoretical calculations are also compared with the available experimental data. The conclusions will be provided in Sec. IV.

\section{METHODS}

In this work three different techniques; namely, the AGS equations, the $\mathrm{HH}$ method, and the FY equations, will be employed to solve the $4 N$ scattering problem and the results provided by the three approaches will be benchmarked. Generalities common to the three methods will be discussed in this section, whereas technicalities proper to each technique will be presented separately in devoted sections.

In the case of a two-body clustering $A-B$, the total energy of the scattering state in the center-of-mass (c.m.) system is given by

$$
E=-B_{A}-B_{B}+T_{\text {c.m. }},
$$

where

$$
T_{\mathrm{c} . \mathrm{m} .}=\frac{q_{\gamma}^{2}}{2 \mu_{\gamma}}, \quad \frac{1}{\mu_{\gamma}}=\frac{1}{M_{A}}+\frac{1}{M_{B}},
$$

$q_{\gamma}$ is the relative momentum between clusters, and $M_{X}\left(B_{X}\right)$ is the mass (binding energy) of the cluster $X$. Clearly, in the case of a single nucleon $M_{X}=M_{N}$, where $M_{N}$ is the nucleon mass and $B_{X}=0$. In this paper, we limit ourselves to study the scattering for $-B^{3} \mathrm{He}<E<-2 B_{d}$, where $B_{d}$ is the deuteron binding energy. Namely, we consider $4 N$ scattering when the channels $p-{ }^{3} \mathrm{H}$ and $n-{ }^{3} \mathrm{He}$ are open, but the $d-d$ channel is closed.

In the following, $\gamma$ will denote the particular asymptotic clustering $A-B$. More specifically, $\gamma=1$ (2) will correspond to the $p-{ }^{3} \mathrm{H}\left(n-{ }^{3} \mathrm{He}\right)$ asymptotic clustering. Moreover, for example, when discussing $n-{ }^{3} \mathrm{He}$ scattering, the observables will be calculated at a given neutron laboratory energy $E_{n}$, corresponding approximately to $E_{n} \approx(4 / 3) T_{\text {c.m. }}$.

For a given total angular-momentum quantum number $J$ and parity $\pi$, the information on the scattering observables is contained in the $S$ matrix $\mathcal{S}_{\gamma L S, \gamma^{\prime} L^{\prime} S^{\prime}}^{J \pi}$, where $\gamma L S\left(\gamma^{\prime} L^{\prime} S^{\prime}\right)$ denotes the initial (final) clustering type, relative orbital momentum, and channel spin of the two clusters, respectively (see below). Each submatrix of the $S$ matrix representing a separate cluster is clearly no longer unitary. For example, the submatrix describing $n-{ }^{3} \mathrm{He}$ elastic scattering will be denoted as $\mathcal{S}_{\gamma=2 L S, \gamma^{\prime}=2 L^{\prime} S^{\prime}}^{J \pi}(E) \equiv \mathcal{S}_{L S, L^{\prime} S^{\prime}}^{n-^{3} \mathrm{He}, J \pi}(E)$. For the $J^{\pi}=$ $0^{ \pm}$channels, submatrix $\mathcal{S}^{n-{ }^{3} \mathrm{He}, J \pi}$ is of dimension one and can be parametrized in the standard way:

$$
\mathcal{S}_{L S, L S}^{n-{ }^{3} \mathrm{He}, J \pi}=\eta_{L S}^{J \pi} \exp \left(2 i \delta_{L S}^{J \pi}\right)
$$

For the other cases, $\mathcal{S}^{n-{ }^{3} \mathrm{He}, J \pi}$ is of dimension two and is conveniently parametrized as

$$
\mathcal{S}^{n-{ }^{3} \mathrm{He}, J \pi}=\left(\begin{array}{cc}
a & b+i c \\
-(b+i c) & a
\end{array}\right)^{-1}\left(\begin{array}{cc}
\eta_{L S}^{J \pi} \exp \left(2 i \delta_{L S}^{J \pi}\right) & 0 \\
0 & \eta_{L^{\prime} S^{\prime}}^{J \pi} \exp \left(2 i \delta_{L^{\prime} S^{\prime}}^{J \pi}\right)
\end{array}\right)\left(\begin{array}{cc}
a & b+i c \\
-(b+i c) & a
\end{array}\right)
$$


where the (eigen)phase shifts $\delta_{L S}^{J \pi}$, the (eigen) inelasticity parameters $\eta_{L S}^{J \pi}$, and the parameters $a, b$, and $c$ are real (and $\left.a^{2}+b^{2}+c^{2}=1\right)$. Explicitly, $(a, b+i c)$ and $(-b-i c, a)$ are the (right) eigenvectors of the matrix $\mathcal{S}^{n-{ }^{3} \mathrm{He}, J \pi}$ associated with the two eigenvalues, the latter written as $\eta_{L S}^{J \pi} \exp \left(2 i \delta_{L S}^{J \pi}\right)$ and $\eta_{L^{\prime} S^{\prime}}^{J \pi} \exp \left(2 i \delta_{L^{\prime} S^{\prime}}^{J \pi}\right)$. Next, we parametrize $a+i(b+i c) \equiv$ $\exp \left(i \epsilon^{J \pi}\right)$. If the matrix $\mathcal{S}^{n-{ }^{3} \mathrm{He}, J \pi}$ would be unitary, one recovers the standard definition of mixing parameter $a=$ $\cos \left(\epsilon^{J \pi}\right), b=\sin \left(\epsilon^{J \pi}\right), c=0$ with $\epsilon^{J \pi}$ real. On the other hand, with inelastic channels present, $\epsilon^{J \pi}$ is complex.

\section{A. Alt-Grassberger-Sandhas equations}

This method is completely taken over from Ref. [16], hence we provide here only a short description. The AGS equations [13] for the four-body transition operators were derived by assuming short-range interactions, but together with the screening and renormalization method $[15,17,18]$, they can be applied also to systems with repulsive Coulomb force. The isospin formalism enables the symmetrization of the AGS equations [14] in the $4 N$ system, where there are only two distinct four-particle partitions, one of the $3+1$ type and one of the $2+2$ type, denoted by $\alpha=1$ and 2 , respectively. In terms of particles $1,2,3,4$ we choose those partitions to be $(12,3) 4$ and $(12)(34)$, respectively. The corresponding transition operators $\mathcal{U}_{\beta \alpha}$ for the initial states of the $3+1$ type, as appropriate for the $n-{ }^{3} \mathrm{He}$ and $p-{ }^{3} \mathrm{H}$ scattering, obey the integral equations

$$
\mathcal{U}_{11}=-\left(G_{0} T G_{0}\right)^{-1} P_{34}-P_{34} U_{1} G_{0} T G_{0} \mathcal{U}_{11}+U_{2} G_{0} T G_{0} \mathcal{U}_{21}
$$

$$
\mathcal{U}_{21}=\left(G_{0} T G_{0}\right)^{-1}\left(1-P_{34}\right)+\left(1-P_{34}\right) U_{1} G_{0} T G_{0} \mathcal{U}_{11} .
$$

Here $G_{0}=\left(E+i 0-H_{0}\right)^{-1}$ is the free resolvent, $H_{0}$ is the free Hamiltonian, $P_{i j}$ is the permutation operator of particles $i$ and $j, T=V+V G_{0} T$ is the two-nucleon transition matrix derived from the potential $V$, and

$$
U_{\alpha}=P_{\alpha} G_{0}^{-1}+P_{\alpha} T G_{0} U_{\alpha}
$$

are $3+1$ and $2+2$ subsystem transition operators with $P_{1}=$ $P_{12} P_{23}+P_{13} P_{23}$ and $P_{2}=P_{13} P_{24}$.

The integral AGS equations (5) are solved in a momentumspace partial-wave framework. Note that in the considered energy regime the only singularities in the kernel of the AGS equations arise due to bound-state poles of $U_{1}$ and are treated by a simple subtraction method. Scattering amplitudes for elastic and charge-exchange reactions are given by on-shell matrix elements of $\mathcal{U}_{11}$ as described in Refs. [14,16,33], where also further details regarding the numerical solution can be found. For example, the $S$ matrix for elastic $n-{ }^{3} \mathrm{He}$ scattering is given by

$$
\mathcal{S}_{L S, L^{\prime} S^{\prime}}^{n-{ }^{3} \mathrm{He}, J \pi}(E)=\delta_{L L^{\prime}} \delta_{S S^{\prime}}-2 \pi i \mu_{2} q_{2} 3\left\langle\phi_{L S J \pi}^{n-{ }^{3} \mathrm{He}}\left|\mathcal{U}_{11}\right| \phi_{L^{\prime} S^{\prime} J \pi}^{n{ }^{3} \mathrm{He}}\right\rangle,
$$

where $\left|\phi_{L S J \pi}^{n-{ }^{3} \mathrm{He}}\right\rangle$ is the Faddeev component of the asymptotic $n-{ }^{3} \mathrm{He}$ state. To obtain fully converged results we include $4 \mathrm{~N}$ states with Jacobi orbital angular momenta $l_{x}, l_{y}, l_{z} \leqslant l_{i}^{\max }=7$
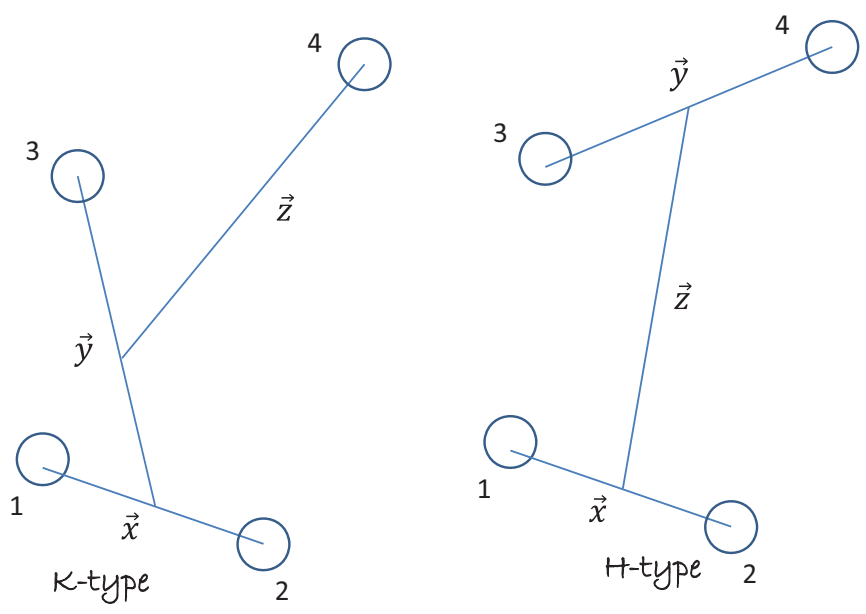

FIG. 1. FY components $K$ and $H$. Asymptotically as $z \rightarrow \infty$ components $K$ describe $3+1$ particle channels, whereas components $H$ contains asymptotic states of $2+2$ channels.

(see also Fig. 1); these cutoffs are higher than in other methods due to the treatment of the Coulomb force through screening and renormalization. The results are also very well converged with respect to the number of discretization grid points, e.g., the accuracy for most $n-{ }^{3} \mathrm{He}$ inelasticity parameters and phase shifts is better than 0.003 and 0.1 degrees, respectively. The only exception are $J^{\pi}=0^{ \pm}$partial waves where the presence of the $4 N$ resonant states near threshold limits the accuracy of our iterative solution method. Typically, theoretical errors for $\eta_{00}^{0+}\left(\eta_{11}^{0-}\right)$ and $\delta_{00}^{0+}\left(\delta_{11}^{0-}\right)$ are $0.01(0.003)$ and $0.5(0.15)$ degrees, respectively. Nevertheless, these small uncertainties have negligible effect on scattering observables, since the corresponding errors do not exceed the width of the curves in the plots shown in Sec. III.

\section{B. Hyperspherical harmonics expansion}

The wave function describing a scattering process with incoming clusters specified by the index $\gamma$ and in a state of total angular-momentum quantum numbers $J, J_{z}$, relative orbital angular momentum $L$, and channel spin $S(S=0,1)$ can be written as

$$
\Psi_{1+3}^{\gamma L S, J J_{z}}=\Psi_{C}^{\gamma L S, J J_{z}}+\Psi_{A}^{\gamma L S, J J_{z}},
$$

keeping in mind the notation $\gamma=1$ (2) to represent respectively $p-{ }^{3} \mathrm{H}\left(n-{ }^{3} \mathrm{He}\right)$ asymptotic clustering.

The "core" part of the wave function $\Psi_{C}^{\gamma L S, J J_{z}}$ describes the system in the region where four particles are close to each other and where their mutual interactions are strong. Hence, $\Psi_{C}^{\gamma L S, J J_{z}}$ vanishes in the limit of large intercluster distances. This part of the wave function is written as a linear expansion $\sum_{\mu} c_{\mu}^{\gamma L S J} \mathcal{Y}_{\mu}$, where $\mathcal{Y}_{\mu}$ is a set of basis functions constructed in terms of the $\mathrm{HH}$ functions (for more details see, for example, Ref. [24]).

The other part $\Psi_{A}^{\gamma L S, J J_{z}}$ describes the relative motion of the clusters in the asymptotic regions, where these clusters do not interact (except eventually for the long-range Coulomb interaction). In the asymptotic region the wave function 
$\Psi_{1+3}^{\gamma L S, J J_{z}}$ reduces to $\Psi_{A}^{\gamma L S, J J_{z}}$, which must therefore be the appropriate asymptotic solution of the Schrödinger equation. Then, $\Psi_{A}^{\gamma L S, J J_{z}}$ can be decomposed into a linear combination of the following functions:

$$
\begin{aligned}
\Omega_{\gamma L S}^{ \pm}= & \mathcal{A}\left\{\left[Y_{L}\left(\hat{\boldsymbol{y}}_{\gamma}\right) \otimes\left[\phi_{A} \otimes \phi_{B}\right]_{S}\right]_{J J_{z}}\right. \\
& \left.\times\left(f_{L}\left(y_{l}\right) \frac{G_{L}\left(\eta_{\gamma}, q_{\gamma} y_{\gamma}\right)}{q_{\gamma} y_{\gamma}} \pm i \frac{F_{L}\left(\eta_{\gamma}, q_{\gamma} y_{\gamma}\right)}{q_{\gamma} y_{\gamma}}\right)\right\}
\end{aligned}
$$

where $y_{\gamma}$ is the distance between the c.m. of clusters $A$ and $B, q_{\gamma}$ is the magnitude of the relative momentum between the two clusters [see Eq. (2)], and $\phi_{A}$ and $\phi_{B}$ the corresponding bound-state wave functions. In the present work, the trinucleon bound-state wave functions are calculated very accurately by means of the $\mathrm{HH}$ method [10,24] using the corresponding $A=3$ Hamiltonian. Conventionally, we identify the trinucleon bound-state wave function with $\phi_{A}$. Therefore, $\phi_{B}$ describes the single nucleon spin-isospin state. The channel spin $S$ is obtained by coupling the angular momentum of the two clusters. In our case, clearly $S=0,1$. The symbol $\mathcal{A}$ means that the expression between the curly braces has to be properly antisymmetrized.

In Eq. (10), the functions $F_{L}$ and $G_{L}$ describe the asymptotic radial motion of the clusters $A$ and $B$. If the two clusters are composed of $Z_{A}$ and $Z_{B}$ protons, respectively, the parameter $\eta_{\gamma}=\mu_{\gamma} Z_{A} Z_{B} e^{2} / q_{\gamma}$, where $e^{2} \approx 1.44 \mathrm{MeV}$ fm. The function $F_{L}(\eta, q y)$ is the regular Coulomb function while $G_{L}(\eta, q y)$ is the irregular Coulomb function. The function $f_{L}(y)=[1-\exp (-\beta y)]^{2 L+1}$ in Eq. (10) has been introduced to regularize $G_{L}$ at small $y$, and $f_{L}(y) \rightarrow 1$ as $y$ is large, thus not affecting the asymptotic behavior of $\Psi_{1+3}^{\gamma L S, J J_{z}}$. Note that, for large values of $q y_{l}$,

$$
\begin{aligned}
& f_{L}\left(y_{l}\right) G_{L}\left(\eta, q y_{l}\right) \pm i F_{L}\left(\eta, q y_{l}\right) \\
& \quad \rightarrow \exp \left[ \pm i\left(q y_{l}-L \pi / 2-\eta \ln \left(2 q y_{l}\right)+\sigma_{L}\right)\right]
\end{aligned}
$$

where $\sigma_{L}$ is the Coulomb phase shift. If $\eta$ is zero, the Coulomb functions reduce to the Riccati-Bessel functions [67]. Therefore, $\Omega_{\gamma L S, J J_{z}}^{+}\left(\Omega_{\gamma L S, J J_{z}}^{-}\right)$describe the asymptotic outgoing (incoming) $A-B$ relative motion. Finally, $\Psi_{A}^{\gamma L S, J J_{z}}$ is given by

$$
\Psi_{A}^{\gamma L S, J J_{z}}=\Omega_{\gamma L S, J J_{z}}^{-}-\sum_{\gamma^{\prime} L^{\prime} S^{\prime}} \mathcal{S}_{\gamma L S, \gamma^{\prime} L^{\prime} S^{\prime}}^{J \pi}(E) \Omega_{\gamma^{\prime} L^{\prime} S^{\prime}, J J_{z}}^{+},
$$

where the parameters $\mathcal{S}_{\gamma L S, \gamma^{\prime} L^{\prime} S^{\prime}}^{J \pi}(E)$ are the $S$-matrix elements at the energy $E$, given by Eq. (1). Of course, the sum over $L^{\prime}$ and $S^{\prime}$ is over all values compatible with the given $J$ and parity $\pi$. In particular, the sum over $L^{\prime}$ is limited to include either even or odd values such that $(-1)^{L^{\prime}}=(-1)^{L}=\pi$. The sum over $\gamma^{\prime}$ is limited to the open channels [namely, those channels for which $q_{\gamma}^{2}>0$, see Eq. (2)]. For the scattering process considered in the present paper, clearly $\gamma^{\prime}=1,2$.

The $S$-matrix elements $\mathcal{S}_{\gamma L S, \gamma^{\prime} L^{\prime} S^{\prime}}^{J \pi}(E)$ and coefficients $c_{\mu}^{\gamma L S J}$ occurring in the $\mathrm{HH}$ expansion of $\Psi_{C}^{\gamma L S, J J_{z}}$ are deter- mined by forming a functional

$$
\begin{aligned}
{\left[\mathcal{S}_{\gamma L S, \gamma^{\prime} L^{\prime} S^{\prime}}^{J \pi}(E)\right]=} & \mathcal{S}_{\gamma L S, \gamma^{\prime} L^{\prime} S^{\prime}}^{J \pi}(E) \\
& -\frac{1}{2 i}\left\langle\Psi_{1+3}^{\gamma^{\prime} L^{\prime} S^{\prime}, J J_{z}}|H-E| \Psi_{1+3}^{\gamma L S, J J_{z}}\right\rangle
\end{aligned}
$$

stationary with respect to variations in $\mathcal{S}_{\gamma L S, \gamma^{\prime} L^{\prime} S^{\prime}}^{J \pi}$ and $c_{\mu}^{\gamma L S J}$ (Kohn variational principle). By applying this principle, a linear set of equations is obtained for $\mathcal{S}_{\gamma L S, \gamma^{\prime} L^{\prime} S^{\prime}}^{J \pi}$ and $c_{\mu}^{\gamma L S J}$. This linear system is solved using the Lanczos algorithm.

This method can be applied in either coordinate or momentum space, and using either local or nonlocal potentials [24]. The first steps are (1) the use of the method discussed in Ref. [68] to antisymmetrize the $\mathrm{HH}$ functions and (2) a partial-wave decomposition of the asymptotic functions $\Omega_{\gamma L S, J J_{z}}^{ \pm}$, the latter task being rather time consuming. After this decomposition, the calculation of the matrix element in Eq. (13) is fast, except for the $J^{\pi}=2^{-}$state, due to the large number of $\mathrm{HH}$ functions to be included in the expansion in this particular case. After these steps, the problem reduces to the solution of a linear system.

The expansion of the scattering wave function in terms of the $\mathrm{HH}$ basis is in principle infinite, therefore a truncation scheme is necessary. The $\mathrm{HH}$ functions are essentially characterized by the orbital angular-momentum quantum numbers $\ell_{i}, i=1,3$, associated with the three Jacobi vectors, and the grand angular quantum number $K$ (each $\mathrm{HH}$ function is a polynomial of degree $K$ ). The basis is truncated to include states with $\ell_{1}+\ell_{2}+\ell_{3} \leqslant \ell_{\max }$ (with all possible recoupling between angular and spin states appropriate to the given $J$ ). Between these states, we retain only the $\mathrm{HH}$ functions with $K \leqslant K_{\max }$. Note that in the calculation we included both states with total isospin $T=0$ and 1 .

The main sources of numerical uncertainties for this method could come from the numerical integration needed to compute the matrix elements of the Hamiltonian and the truncation of the basis. It has been checked that the numerical uncertainty of the calculated phase-shifts related to the numerical integration is small (around $0.1 \%$ ). The $N N$ interaction has been limited to act on two-body states with total angular momentum $j \leqslant j_{\max }=8$ (greater values of $j_{\max }$ are completely negligible). The largest uncertainty is related to the use of a finite basis due to the slow convergence of the results with $K_{\max }$. This problem can be partially overcome by performing calculations for increasing values of $K_{\max }$ and then using some extrapolation rule (see, for example, Ref. [69]) to get the " $K_{\max } \rightarrow \infty$ " result. This procedure introduces a new uncertainty which can be estimated. A detailed study of this problem will be published elsewhere [70]. The convergence of the quantities of interest in term of $K_{\max }$ is slower using $N N$ potentials with a strong repulsion at short interparticle distance, but it is less relevant for the N3LO500 potential. For the present case, this uncertainty has been estimated to be at most $0.5 \%$.

The convergence with $\ell_{\max }$ is usually rather fast and values of $\ell_{\max }$ around either 5 or 6 have been found to be sufficient. However, in some cases, we have found a slow convergence of the inelasticity parameters $\eta_{L S}^{J \pi}$. To give an example, in 
TABLE I. $n-{ }^{3} \mathrm{He}$ inelasticity parameter $\eta_{11}^{0-}$ and phase shift $\delta_{11}^{0-}$ the $J^{\pi}=0^{-}$wave at $E_{n}=1.0 \mathrm{MeV}$, obtained with the $\mathrm{HH}$ method as a function of $\ell_{\max }$ as well as for the AGS and FY methods as a function of $l_{i}^{\max }$ (see text for details). The calculations have been performed by using the N3LO500 potential.

\begin{tabular}{|c|c|c|c|c|}
\hline$\ell_{\max }(\mathrm{HH})$ & $\eta_{11}^{0-}$ & $\delta_{11}^{0-}(\mathrm{deg})$ & & \\
\hline 1 & 0.603 & +21.9 & & \\
\hline 3 & 0.459 & -9.9 & & \\
\hline 5 & 0.505 & -10.5 & & \\
\hline \multirow[t]{2}{*}{$l_{i}^{\max }(\mathrm{AGS} / \mathrm{FY})$} & \multicolumn{2}{|c|}{ AGS } & \multicolumn{2}{|c|}{ FY } \\
\hline & $\eta_{11}^{0-}$ & $\delta_{11}^{0-}(\operatorname{deg})$ & $\eta_{11}^{0-}$ & $\delta_{11}^{0-}(\mathrm{deg})$ \\
\hline 3 & 0.544 & -10.44 & 0.538 & -10.14 \\
\hline 4 & 0.550 & -10.47 & 0.545 & -10.38 \\
\hline$\geqslant 5$ & 0.551 & -10.47 & 0.547 & -10.36 \\
\hline
\end{tabular}

Table I, we report the values of the $n-{ }^{3} \mathrm{He} \eta_{L S}^{J \pi}$ and $\delta_{L S}^{J \pi}$ parameters for the $J^{\pi}=0^{-}$wave, calculated with the $\mathrm{HH}$ method as a function of $\ell_{\max }$. The calculations have been performed at $E_{n}=1 \mathrm{MeV}$ and for the N3LO500 potential. For this wave the parity is negative, so only $\mathrm{HH}$ functions having $\ell_{\max }$ odd have to be included in the expansion. As can be seen, the inclusion in the expansion of the "core" part of functions with $\ell_{\max }=1$ is insufficient to obtain a reasonable estimate of these parameters. The addition also of the $\ell_{\max }=3$ functions improves noticeably the calculation of the phase shift $\delta_{11}^{0-}$, which is now very close to the final result (in the table, we have also reported the same parameters obtained by using the AGS equations). The inclusion of $\ell_{\max }=5$ brings finally the $\mathrm{HH}$ results in agreement with that obtained by the AGS method. On the other hand, the $\eta_{11}^{0-}$ values appears to converge slowly. Note that for $p-{ }^{3} \mathrm{He}$ scattering (see our previous benchmark [32]), only one asymptotic channel is open, so in all calculations one obtains $\eta_{11}^{0-}=1$ with a very good accuracy.

\section{Faddeev-Yakubovsky equations in configuration space}

In late sixties Yakubovsky [71,72] generalized a set of equations proposed by Faddeev [71], to treat scattering problems beyond the $A=3$ case. Based on the arithmetic properties, which arise from the subsequent breaking of an $N$-particle system into its subclusters, FY formalism offers a natural way to decompose a system's wave function into the so-called Faddeev-Yakubovsky components (FYCs). As a result, FYCs represent the natural structures to impose a proper wave function behavior at the boundaries. A fourparticle system requires us to introduce FYCs of two distinct types: components $K$ and $H$. Asymptotes of components $K$ incorporate $3+1$ particle channels, while components $H$ contain asymptotes of $2+2$ particle channels (see Fig. 1). By interchanging the order of particles one can construct twelve different components of type $K$ and six of type $H$. The system wave function is then represented simply as a sum of these 18 FYCs.

Employing the formalism of isospin, protons and neutrons become two distinct states of the same particle (nucleon). For a system of four identical particles there exist only two independent FYCs, one of type $K$ and one of type $H$. The other 16 FYCs can be obtained from the two independent FYCs by applying particle permutation operators (i.e., interchanging the order of particles in the system). Similarly, only two independent FY equations exist; by singling out $K \equiv K_{1,23}^{4}$ and $H \equiv H_{12}^{34}$, the set of two FY equations read [20,22]

$$
\begin{aligned}
& \left(E-H_{0}-V_{12}\right) K=V_{12}\left(P^{+}+P^{-}\right)[(1+Q) K+H], \\
& \left(E-H_{0}-V_{12}\right) H=V_{12} \tilde{P}[(1+Q) K+H] .
\end{aligned}
$$

The particle permutation operators $P^{+}, P^{-}, \tilde{P}$, and $Q$ represent simply different combinations of two-particle transposition operators:

$$
\begin{aligned}
P^{+} & =\left(P^{-}\right)^{-1}=P_{23} P_{12}, \\
Q & =-P_{34}, \\
\tilde{P} & =P_{13} P_{24}=P_{24} P_{13} .
\end{aligned}
$$

These permutation operators are in fact the same as used in the AGS method, since $P_{1}=P^{+}+P^{-}$and $P_{2}=\tilde{P}$. Employing the operators defined above, the wave function of the system is given by

$$
\begin{aligned}
\Psi= & {\left[1+\left(1+P^{+}+P^{-}\right) Q\right]\left(1+P^{+}+P^{-}\right) K } \\
& +\left(1+P^{+}+P^{-}\right)(1+\tilde{P}) H .
\end{aligned}
$$

The functions $K$ and $H$ are expanded in the basis of partial angular momentum, spin, and isospin variables, according to

$$
\Phi_{i}\left(\vec{x}_{i}, \vec{y}_{i}, \vec{z}_{i}\right)=\sum_{\alpha} \frac{\mathcal{F}_{i}^{\alpha}\left(x_{i}, y_{i}, z_{i}\right)}{x_{i} y_{i} z_{i}} Y_{i}^{\alpha}\left(\hat{x}_{i}, \hat{y}_{i}, \hat{z}_{i}\right),
$$

where the functions $Y_{i}^{\alpha}\left(\hat{x}_{i}, \hat{y}_{i}, \hat{z}_{i}\right)$ are defined below. The Jacobi coordinates, associated with each type of FYC $K$ and $H$, are used to represent our wave functions (see Fig. 1). Such a choice of coordinates permits us to separate the center-of-mass motion and guarantees that the kinetic-energy operator is independent of angular variables. The angular dependence is hidden in tripolar harmonics $Y_{i}^{\alpha}\left(\hat{x}_{i}, \hat{y}_{i}, \hat{z}_{i}\right)$, which in addition to angular-momentum variables comprise spins and isospins of the nucleons. To couple the angular and spin quantum numbers, a slightly different scheme is employed compared with AGS and HH methods; namely, the $j j$-coupling scheme, which is defined by

$$
\left[\left\{\left(t_{i} t_{j}\right)_{t_{x}} t_{k}\right\}_{T_{3}} t_{l}\right]_{T T_{z}}\left\langle\left\{\left[l_{x}\left(s_{i} s_{j}\right)_{\sigma_{x}}\right]_{j_{x}}\left[l_{y} s_{k}\right]_{j_{y}}\right\}_{J_{3}}\left[l_{z} s_{l}\right]_{j_{z}}\right\rangle_{J^{\pi} J_{z}}
$$

for the components of type $K$, and

$$
\left[\left(t_{i} t_{j}\right)_{t_{x}}\left(t_{k} t_{l}\right)_{t_{y}}\right]_{T T_{z}}\left\langle\left\{\left[l_{x}\left(s_{i} s_{j}\right)_{\sigma_{x}}\right]_{j_{x}}\left[l_{y}\left(s_{k} s_{l}\right)_{\sigma_{y}}\right]_{j_{y}}\right\}_{j_{x y}} l_{z}\right\rangle_{J^{\pi} J_{z}}
$$

for the $H$-type components. Here $s_{i}=1 / 2$ and $t_{i}=1 / 2$ are the spin and isospin quantum numbers of the individual nucleons and $\left(J^{\pi}, T\right)$ are, respectively, the total angular momentum, parity, and isospin of a four-body system. By $J_{z}$ and $T_{z}$ we identify the projection of the system total angular momentum and isospin on the selected axis. The nuclear Hamiltonian conserves the system parity and angular momentum $J^{\pi}$; the system wave function is also invariant for the rotations around the fixed axis (so one can also fix projection quantum number $J_{z}$ ). Furthermore the system made of two protons and two neutrons has $T_{z} \equiv 0$. Then each amplitude $\mathcal{F}_{i}^{\alpha}\left(x_{i}, y_{i}, z_{i}\right)$ is 
labeled by a set of 11 nonfixed quantum numbers $\alpha$. On the contrary, the total isospin $T$ of the system is not conserved, it is allowed to take values $T=0,1$, and 2. Combination of different total isospin channels is necessary in order to separate unambiguously the $p-{ }^{3} \mathrm{H}$ and $n-{ }^{3} \mathrm{He}$ channels [20].

By projecting each of Eqs. (14) on its natural configuration space basis, one obtains a system of coupled integrodifferential equations. To keep the number of these equations finite, one is obliged to introduce some additional truncations in the partial-wave expansion given in Eq. (16), by considering only the most relevant amplitudes. This truncation is realized by imposing the condition $\max \left(l_{x}, l_{y}, l_{z}\right) \leqslant 4$ on the maximal partial angular momenta.

Equations (14) are not complete as long as they are not supplemented with the appropriate boundary conditions. First, FY amplitudes, for bound as well as for scattering states, satisfy the regularity conditions:

$$
\mathcal{F}_{i}^{\alpha}\left(0, y_{i}, z_{i}\right)=\mathcal{F}_{i}^{\alpha}\left(x_{i}, 0, z_{i}\right)=\mathcal{F}_{i}^{\alpha}\left(x_{i}, y_{i}, 0\right)=0 .
$$

The proper asymptotic behavior of the FY components of type $K$ for the scattering process is implemented in a similar way as for the HH method; see Eq. (9), i.e., by splitting the FY amplitude into two terms: a square integrable core-term $\mathcal{F}_{C, i}^{\alpha}\left(x_{i}, y_{i}, z_{i}\right)$ and a long-ranged term $\mathcal{F}_{A, i}^{\alpha}\left(x_{i}, y_{i}, z_{i}\right)$, which describes the behavior of FY amplitudes in the far asymptotic regions,

$$
\mathcal{F}_{i}^{\alpha}\left(x_{i}, y_{i}, z_{i}\right)=\mathcal{F}_{C, i}^{\alpha}\left(x_{i}, y_{i}, z_{i}\right)+\mathcal{F}_{A, i}^{\alpha}\left(x_{i}, y_{i}, z_{i}\right) .
$$

As explained in the previous section, the asymptotic part $\mathcal{F}_{A, i}^{\alpha}\left(x_{i}, y_{i}, z_{i}\right)$ is constructed from the calculated three-nucleon wave functions (either ${ }^{3} \mathrm{H}$ or ${ }^{3} \mathrm{He}$ nucleus) and involves a few parameters associated with a scattering matrix to be determined; see Eq. (12). This term is treated as inhomogeneous when solving numerically the FY equations. The core part of the FY partial amplitudes is expanded on the basis of Lagrange-Laguerre mesh functions, employing the Lagrange-mesh method [73]:

$$
\mathcal{F}_{C, i}^{\alpha}\left(x_{i}, y_{i}, z_{i}\right)=C^{\alpha, k, l, m} f_{k}^{x}\left(x_{i}\right) f_{l}^{y}\left(y_{i}\right) f_{m}^{z}\left(z_{i}\right),
$$

with $C^{\alpha, k, l, m}$ representing some unknown coefficients, while $f_{k}^{x}\left(x_{i}\right), f_{l}^{y}\left(y_{i}\right)$, and $f_{m}^{z}\left(z_{i}\right)$ are Lagrange-Laguerre basis functions associated with each radial variable. The set of integro-differential equations is transformed into a linear algebra problem by projecting these equations on a chosen three-dimensional Lagrange-Laguerre basis. The coefficients $C^{\alpha, k, l, m}$ are determined by solving the resulting linear algebra problem and by applying the Kohn-variational principle to determine the scattering matrix associated with the inhomogeneous terms of Eq. (20).

Convergence and accuracy of the FY method is similar to those of the AGS one, which allows us to keep the corresponding errors within the width of the curves in the plots shown in Sec. III. Truncation of the partial angular momentum basis to $\max \left(l_{x}, l_{y}, l_{z}\right) \leqslant 4$ guarantees convergence for the phase shifts and for the inelasticity parameters at the level of $\sim 0.2 \%$ and $0.4 \%$, respectively. A typical PW convergence pattern is provided in Table I. Similar uncertainty is due to the Lagrange-Laguerre basis expansion, used to discretize radial dependence of the FY components. The strongest uncertainty is obtained for the mixing parameters $\epsilon^{J \pi}$, when these values are close to zero. This turns to be an artifact of the $j j$-coupling scheme employed together with the FY method: small values of the mixing parameters $\epsilon^{J \pi}$ are directly related with small off-diagonal matrix elements in the $l s$-coupling scheme, while they result from a delicate cancellation of large matrix elements within the $j j$-coupling scheme.

\section{RESULTS}

In this section the results obtained by using the three different methods are compared between themselves as well as with available experimental data for some selected observables. First, in Tables II and III we present the $n-{ }^{3} \mathrm{He}$ phase shifts and inelasticity parameters for the most relevant waves calculated using the three different methods. Calculations have been carried out for three different neutron laboratory energies, $E_{n}=1,2$, and $3.5 \mathrm{MeV}$, corresponding to cases where experimental data exist. In particular, we compare the parameters computed by the three methods for the states

TABLE II. $n-{ }^{3} \mathrm{He}$ inelasticity parameters $\eta_{L S}^{J \pi}$, phase shifts $\delta_{L S}^{J \pi}$, and mixing parameters $\epsilon^{J \pi}$ for the $J^{\pi}=0^{ \pm}$and $1^{+}$waves at $E_{n}=1.0$, 2.0, and 3.5 MeV, obtained with the three methods described in the text. The phase shifts and mixing parameters are given in degrees. The calculations have been performed using the N3LO500 potential.

\begin{tabular}{lccccccccccc}
\hline \hline $\begin{array}{l}E_{n} \\
(\mathrm{MeV})\end{array}$ & $\eta^{0+}$ & $\begin{array}{c}\delta^{0+} \\
(\mathrm{deg})\end{array}$ & $\eta^{0-}$ & $\begin{array}{c}\delta^{0-} \\
(\mathrm{deg})\end{array}$ & $\eta_{01}^{1+}$ & $\begin{array}{c}\delta_{01}^{1+} \\
(\mathrm{deg})\end{array}$ & $\eta_{21}^{1+}$ & $\begin{array}{c}\delta_{21}^{1+} \\
(\mathrm{deg})\end{array}$ & $\begin{array}{c}\operatorname{Re}\left(\epsilon^{1+}\right) \\
(\mathrm{deg})\end{array}$ & $\begin{array}{c}\operatorname{Im}\left(\epsilon^{1+}\right) \\
(\mathrm{deg})\end{array}$ & $\begin{array}{c}\mathrm{Method} \\
1.0\end{array}$ \\
\hline \multirow{2}{*}{2.267} & -63.4 & 0.551 & -10.5 & 0.998 & -31.8 & 1.000 & -0.054 & 0.306 & 0.001 & AGS \\
& 0.267 & -63.2 & 0.545 & -10.4 & 0.997 & -31.8 & 1.000 & -0.058 & 0.228 & 0.001 & FY \\
& 0.259 & -64.0 & 0.505 & -10.5 & 0.998 & -32.4 & 1.000 & -0.065 & 0.297 & 0.001 & HH \\
& 0.162 & -81.4 & 0.380 & -12.9 & 0.997 & -43.8 & 1.000 & -0.220 & 0.585 & 0.003 & AGS \\
& 0.162 & -81.4 & 0.368 & -12.7 & 0.997 & -43.8 & 1.000 & -0.184 & 0.467 & 0.006 & FY \\
& 0.147 & -82.2 & 0.348 & -12.9 & 0.996 & -44.3 & 1.000 & -0.250 & 0.574 & 0.002 & HH \\
3.5 & 0.086 & -97.6 & 0.093 & -8.48 & 0.996 & -56.0 & 1.000 & -0.567 & 0.971 & 0.007 & AGS \\
& 0.086 & -98.2 & 0.075 & -8.41 & 0.996 & -56.3 & 1.000 & -0.566 & 0.884 & 0.003 & FY \\
& 0.074 & -98.5 & 0.088 & -8.16 & 0.996 & -56.2 & 1.000 & -0.618 & 0.957 & 0.004 & HH \\
\hline \hline
\end{tabular}


TABLE III. Same as for Table II but for the $J^{\pi}=1^{-}$and $2^{-}$waves.

\begin{tabular}{|c|c|c|c|c|c|c|c|c|c|c|c|c|c|}
\hline $\begin{array}{l}E_{n} \\
(\mathrm{MeV})\end{array}$ & $\eta_{10}^{1-}$ & $\begin{array}{c}\delta_{10}^{1-} \\
(\mathrm{deg})\end{array}$ & $\eta_{11}^{1-}$ & $\begin{array}{c}\delta_{11}^{0-} \\
(\mathrm{deg})\end{array}$ & $\begin{array}{c}\operatorname{Re}\left(\epsilon^{1-}\right) \\
(\operatorname{deg})\end{array}$ & $\begin{array}{c}\operatorname{Im}\left(\epsilon^{1-}\right) \\
(\operatorname{deg})\end{array}$ & $\eta_{11}^{2-}$ & $\begin{array}{c}\delta_{11}^{2-} \\
(\operatorname{deg})\end{array}$ & $\eta_{31}^{2-}$ & $\begin{array}{c}\delta_{31}^{2-} \\
(\operatorname{deg})\end{array}$ & $\begin{array}{c}\operatorname{Re}\left(\epsilon^{2-}\right) \\
(\operatorname{deg})\end{array}$ & $\begin{array}{c}\operatorname{Im}\left(\epsilon^{2-}\right) \\
(\operatorname{deg})\end{array}$ & Method \\
\hline \multirow[t]{2}{*}{1.0} & 0.959 & -0.263 & 0.994 & 7.43 & -1.98 & -0.869 & 0.923 & 17.5 & 1.000 & 0.003 & -0.249 & -0.093 & AGS \\
\hline & 0.957 & -0.295 & 0.993 & 7.57 & -2.02 & -0.909 & 0.938 & 17.0 & 1.000 & 0.003 & -0.248 & -0.086 & $\mathrm{HH}$ \\
\hline 2.0 & 0.864 & -0.806 & 0.985 & 20.0 & -1.96 & -1.32 & 0.665 & 47.1 & 1.000 & 0.021 & -0.330 & -0.286 & AGS \\
\hline \multirow[t]{3}{*}{3.5} & 0.699 & -2.60 & 0.992 & 38.6 & -1.90 & -1.65 & 0.676 & 70.8 & 1.000 & 0.101 & -0.237 & -0.422 & AGS \\
\hline & 0.694 & -2.65 & 0.990 & 38.1 & -1.87 & -1.75 & 0.681 & 70.4 & 1.000 & 0.078 & -0.255 & -0.415 & FY \\
\hline & 0.694 & -2.56 & 0.994 & 37.3 & -1.87 & -1.75 & 0.714 & 69.1 & 1.000 & 0.092 & -0.259 & -0.408 & $\mathrm{HH}$ \\
\hline
\end{tabular}

$J^{\pi}=0^{ \pm}, 1^{ \pm}$, and $2^{-}$. The scattering in other $J^{\pi}$ states is dominated by the centrifugal barrier and therefore their $S$-matrices only slightly deviates from the unity matrix, while the results are not very sensitive to the interaction and the method used to calculate them. Note that the calculations of the observables has been performed including states up to $J=4$. In all the cases, the wave function contains states of total isospin $T=0$ and 1 .

Clearly the values of these parameters may depend on the adopted choice of the coupling scheme between the spin of the two clusters and the spherical harmonic function $Y_{L}(\boldsymbol{y})$ in the asymptotic functions $\Omega_{L S}^{ \pm}$[see Eq. (10)]. As specified in the previous section, each group has chosen a different coupling scheme. It can be shown, however, that the (eigen)phase-shifts defined as discussed above are coupling-scheme independent; on the contrary, the mixing parameter depends on coupling scheme (however, they are related to each other by some constant factor). In the following we have decided to report the mixing parameters which are proper to the coupling scheme specified in Eq. (10).

In Tables II and III we present the inelasticity parameters, phase shifts, and mixing parameters for $n-{ }^{3} \mathrm{He}$ scattering obtained by using the N3LO500 potential at the selected energies. By inspecting the tables, we notice a reasonable agreement between the results obtained by the three different techniques.

As presented in Table II, for $0^{ \pm}$waves we note a large deviation of the inelasticity parameters from unity. In these waves, a $n-{ }^{3} \mathrm{He}$ initial state will mostly end up in a $p-{ }^{3} \mathrm{H}$ final state (and vice versa). The phase shifts are in very good agreement, only in a few cases do the results differ by more than $1 \%$. Larger differences are found for the inelasticity parameters (up to $10 \%$ ), related with the aforementioned slow convergence for these values within the $\mathrm{HH}$ method. Note that the $n-{ }^{3} \mathrm{He}^{-}$phase shifts are negative, meaning that the effective interaction between the two clusters is mostly repulsive in this wave. This is at variance with the $p-{ }^{3} \mathrm{He}$ scattering case, where it was found that the interaction is attractive for the same wave. Inelasticity parameter for $1^{+}$ wave is found to be close to unity. For this wave, the Pauli repulsion keeps the incident clusters well apart, preventing particle recombination process.
Let us now inspect Table III. For the $1^{-}$state, it is possible to note that the $L S=10\left({ }^{1} P_{1}\right)$ phase shift is negative, showing that the interaction of the $n-{ }^{3} \mathrm{He}$ clusters is repulsive (again, for this wave the $p-{ }^{3} \mathrm{He}$ phase shift is positive). In this case, the inelasticity parameter deviates sizably from unity. On the other hand, the $L S=11\left({ }^{3} P_{1}\right)$ phase shift is positive and large as for $p-{ }^{3} \mathrm{He}$, while $\eta_{11}^{1-} \approx 1$. We note a good agreement between the results obtained by the three different methods for these parameters, and also for the mixing parameter $\epsilon^{1-}$. For the $2^{-}$state, the $L S=11\left({ }^{3} P_{2}\right)$ phase shift is positive and large as for $p-{ }^{3} \mathrm{He}$, while the corresponding inelasticity parameter decreases at low energies and then reaches a sort of plateau between $E_{n}=2$ and $3.5 \mathrm{MeV}$, as confirmed by several calculations performed between these two energies. There is a good agreement for the phase shifts, while for the inelasticity parameters we observe again a somewhat sizable deviation between the results obtained with the $\mathrm{HH}$ and the AGS and FY methods, again connected to the slow convergence of the HH expansion. The $L S=31\left({ }^{3} F_{2}\right)$ phase shift $\delta_{31}^{2-}$ and mixing parameter $\epsilon^{2-}$ are rather small, due to the large centrifugal barrier (in this case, $\eta_{31}^{2-}$ is very close to 1 ). In any case, we observe a reasonable agreement between different calculation methods even for these tiny quantities.

It is important to note that the observed deviations between the results of the three calculations are in part related to the different coupling schemes employed to couple angular and spin degrees of freedom. For example, in the FY calculation a $j j$-coupling scheme is used; see Eqs. (17) and (18), while an $l s$-coupling scheme is used in the HH and AGS methods. In some cases, small off-diagonal matrix elements, obtained straightforwardly in one scheme, come from a delicate cancellation of large matrix elements in the other scheme. This explains the large relative variation observed sometimes for the phase shift and mixing angle parameters. However, the observables are not in general very sensitive to which scheme is used; see below.

Let us now examine how the good agreement found for the phase-shifts and mixing parameters calculated with the three different methods is reflected in the experimental observables. Let us start with a case of $n-{ }^{3} \mathrm{He}$ elastic observables. We have considered the differential cross section, the neutron analyzing power $A_{y 0}$, the ${ }^{3} \mathrm{He}$ analyzing power $A_{0 y}$, and the 


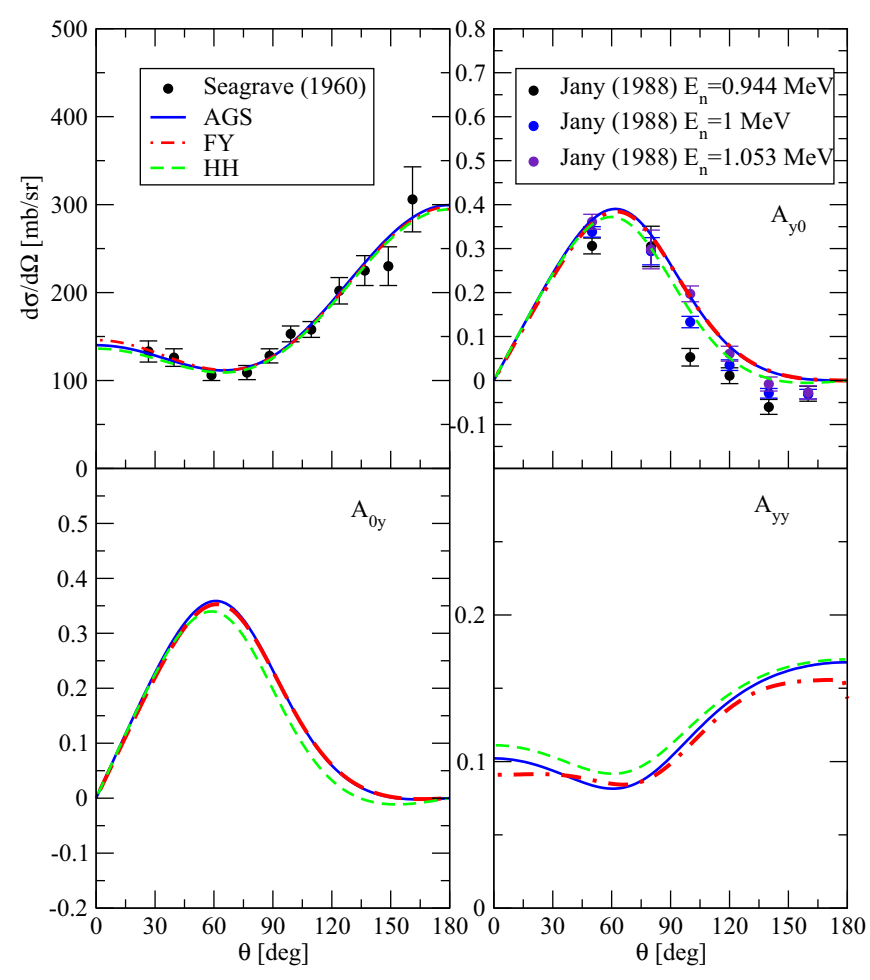

FIG. 2. Differential cross section, proton analyzing power $A_{y 0},{ }^{3} \mathrm{He}$ analyzing power $A_{0 y}$, and spin-correlation coefficient $A_{y y}$ for $n-{ }^{3} \mathrm{He}$ elastic scattering at $E_{n}=1 \mathrm{MeV}$ neutron laboratory energy obtained by using the N3LO500 potential. The lines show the results obtained by using the AGS (blue solid lines), FY (red dot-dash lines), and the $\mathrm{HH}$ (green dashed lines) methods. The experimental data are from Refs. [42,49].

spin-correlation coefficient $A_{y y}$. The analyzing power observables are rather sensitive to small variations of the $P$ wave phase shifts in the kinematical regime considered in this paper, while $A_{y y}$ is also sensitive to the $S$-wave phase shifts.

In Figs. 2, 3, and 4 we report the results obtained by using the AGS equation (blue solid lines), the HH expansion method (green dashed lines), and the FY equations (red dot-dash lines) using the N3LO500 potential for $E_{n}=1,2$, and $3.5 \mathrm{MeV}$, respectively. Where available, we compare the calculated observables with the experimental data. As can be seen by inspecting the three figures, for the differential cross section the three curves almost always perfectly coincide and can be hardly distinguished. For the $A_{y 0}$ and $A_{0 y}$ analyzing power observables, the AGS and FY results almost coincide, while the $\mathrm{HH}$ results slightly differ (however, we note that the differences between the three calculations are in any case within the experimental error bars). We also note in Fig. 2 a rather strong energy dependence of the analyzing power from the measurements at $E_{n}=0.944,1$, and $1.053 \mathrm{MeV}$ [49]. For the $A_{y y}$ observable, the predictions obtained by the three methods are slightly at variance. This observable, as already stated, is quite sensitive to the $0^{+}$phase shift, for which the convergence of the three methods is more problematic. In spite of these difficulties, the agreement in the considered observable is still acceptable.

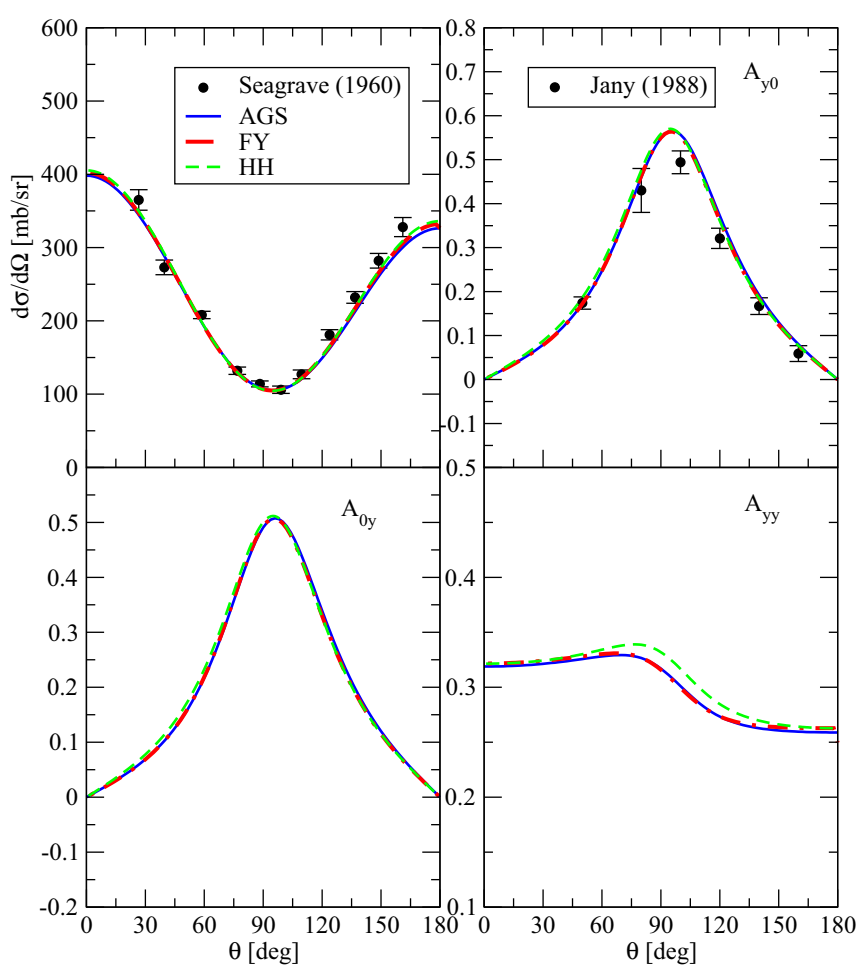

FIG. 3. Same as Fig. 2 but for neutron energy $E_{n}=2 \mathrm{MeV}$. The experimental data are from Refs. [42,49].

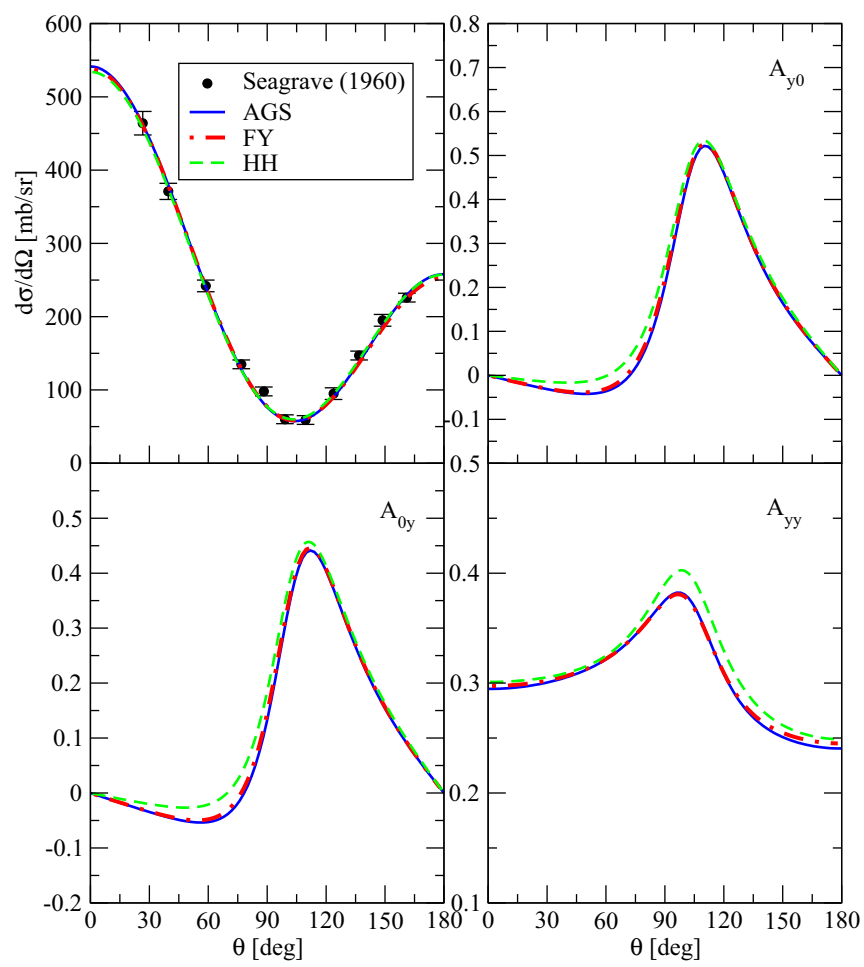

FIG. 4. Same as Fig. 2 but for neutron energy $E_{n}=3.5 \mathrm{MeV}$. The experimental data are from Ref. [42]. 


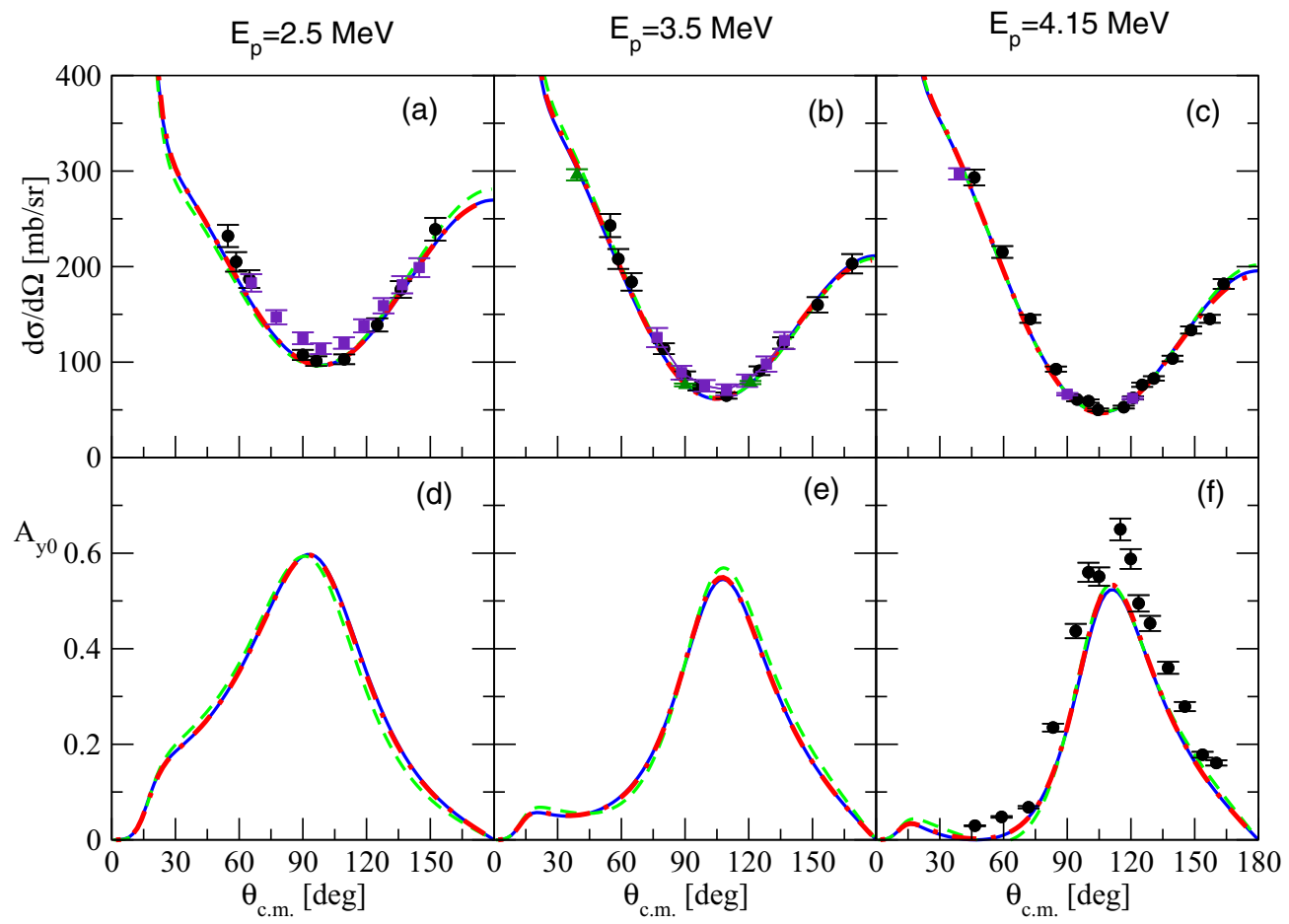

FIG. 5. Differential cross sections (upper panels) and proton analyzing powers $A_{y 0}$ (lower panels) for $p-{ }^{3} \mathrm{H}$ elastic scattering at $E_{p}=$ 2.5, 3.5, and $4.15 \mathrm{MeV}$ proton energies obtained by using the N3LO500 potential. The lines show the results obtained by using the AGS (blue solid lines), FY (red dot-dash lines), and the HH (green dashed lines) methods. In many cases, the curves overlap and cannot be distinguished. The experimental data in panel (a) are from Refs. [36] (circles) and [35] (squares), in panel (b) from Refs. [36] (circles), [39] (squares), and [40] (triangles), in panel (c) from Refs. [41] (circles) and [40] (squares), and finally in panel (f) from Ref. [41] (circles).

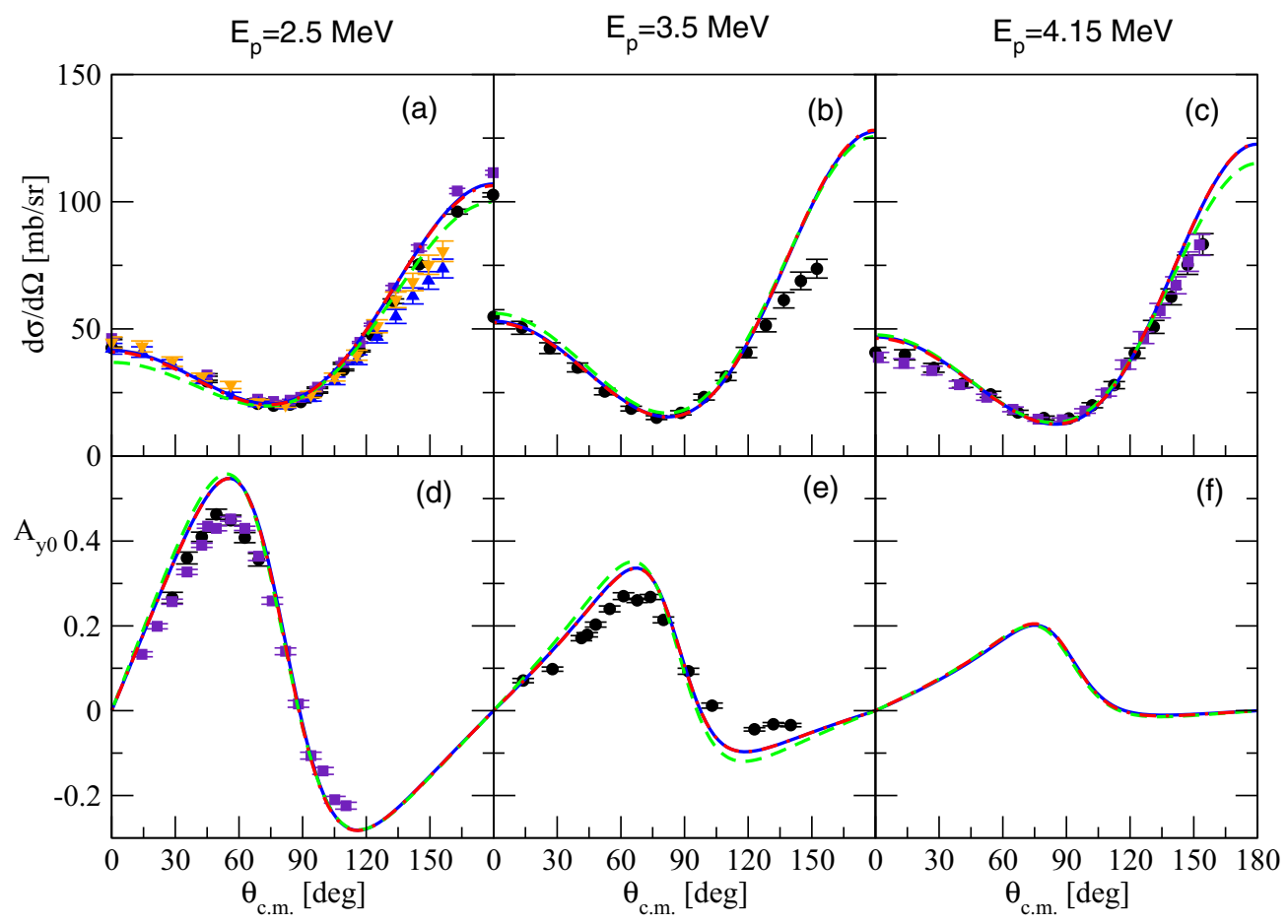

FIG. 6. Same as Fig. 5 but for $p-{ }^{3} \mathrm{H} \rightarrow n-{ }^{3} \mathrm{He}$ process. The experimental data in panel (a) are from Ref. [61] (circles and squares) and Ref. [60] (up and down triangles), in panel (b) from Ref. [60] (circles), in panel (c) from Refs. [60] (circles) and [59] (squares), in panel (d) from Refs. [64] (circles) and [63] (squares), and finally in panel (e) from Ref. [63] (circles). 
Let us now consider the $p-{ }^{3} \mathrm{H}$ elastic observables. For this case, we have decided to show the comparison of the theoretical results for the differential cross section and the proton analyzing power $A_{y 0}$, where experimental data are available. These observables are reported in Fig. 5 for three different energies of the proton beam, $E_{p}=2.5,3.5$, and 4.15 MeV. In the upper panels, we report the differential cross sections and in the lower panels the proton analyzing power $A_{y 0}$. As can be seen, the AGS and FY results are almost indistinguishable for all the cases considered. The HH results show somewhere a slight deviation from the AGS and FY values, probably due to the slow convergence observed for the inelasticity parameters.

Regarding the comparison with the experimental data, we note again a good reproduction of the differential cross sections at all energies. For $A_{y 0}$ at $E_{p}=4.15 \mathrm{MeV}$, the only case for which there are experimental data for this observable, we note a slight underprediction of the theoretical results in the whole angular range for which data are available.

In Fig. 6, the comparison is extended to the reaction $p-{ }^{3} \mathrm{H} \rightarrow n-{ }^{3} \mathrm{He}$. Also in this case, we report the differential cross section and proton analyzing power at $E_{p}=2.5,3.5$, and $4.15 \mathrm{MeV}$. The $p-{ }^{3} \mathrm{H} \rightarrow n-{ }^{3} \mathrm{He}$ differential cross section has been found to depend sizeably on the $2^{-}$wave. From Figs. 6(a)-6(c), it is possible to note a clear discrepancy among the theoretical calculations and the experimental data at backward angles. Regarding $A_{y 0}$, we observe a fair agreement between the theoretical results. Here, we note an overprediction of the calculated $A_{y 0}$ in the maximum region with respect to the available experimental data. At $E_{p}=3.5 \mathrm{MeV}$, see Fig. 6(e), theoretical calculations and data also disagree in the region of the minimum. The origin of these discrepancies is still not clear. Moreover, we note that in Ref. [16], using four realistic $N N$ potentials, a significant sensitivity of charge-exchange observables to the $N N$ force model has been found.

\section{CONCLUSIONS}

In this work, we have studied some low-energy $n-{ }^{3} \mathrm{He}$ and $p-{ }^{3} \mathrm{H}$ elastic and charge-exchange observables by using three different approaches: the HH, AGS, and FY techniques. Very accurate solutions of the $4 N$ scattering problem by using the AGS technique [14-16] were obtained already a few years ago. The long-range Coulomb interaction in this approach is taken into account by using the screening and renormalization method $[17,18]$. In recent years, after adding some additional numerical power, also the accuracy of the calculations performed by using the $\mathrm{HH}$ and FY techniques increased $[23,25,26]$. Therefore, it becomes quite interesting to compare the results obtained by the different methods in order to test their capability to solve the $4 N$ scattering problem. Around five years ago, some of the authors of the present paper presented a very detailed comparison for $p-{ }^{3} \mathrm{He}$ and $n-{ }^{3} \mathrm{H}$ observables [32]. The aim of the present paper is to extend the benchmark to the $n-{ }^{3} \mathrm{He}$ and $p-{ }^{3} \mathrm{H}$ scattering.

Here we have shown that for N3LO500 potential the results obtained by the different techniques are in good agreement. In particular, FY and AGS results are in a very good agreement. The phase shifts, mixing angles, and observables calculated by using the $\mathrm{HH}$ method show some small deviations from those obtained by the AGS and FY techniques. Anyway, the differences are tiny, and usually do not exceed the experimental errors. Therefore, we can conclude that all the considered theoretical methods have reached a rather high level of accuracy in the description of $n-{ }^{3} \mathrm{He}$ and $p-{ }^{3} \mathrm{H}$ elastic and charge-exchange scattering, making comparison with experiment reliable and meaningful.

Concerning the comparison with the experiments, in most of the cases we observe a good agreement between the results obtained by using the N3LO500 potential and the available experimental data. Some disagreements persist for the analyzing power, and for the $p-{ }^{3} \mathrm{H} \rightarrow n-{ }^{3} \mathrm{He}$ differential cross section at backward angles. These observables show also a sizable $N N$ interaction model dependence [16]. Therefore as a paramount test of nuclear interaction models, it will be interesting to explore the effect of the inclusion of a $3 N$ interaction. Using the AGS method, accurate studies of $n-{ }^{3} \mathrm{He}$ elastic scattering observables including the $\Delta$ degrees of freedom, a way to introduce effective $3 N$ forces, have been already reported [50]. Moreover, calculations performed for $p-{ }^{3} \mathrm{He}$ and $n-{ }^{3} \mathrm{H}$ elastic scattering have already shown the size and importance of $T=13 N$ forces [26]. It will be therefore certainly very interesting to test the inclusion of the most modern $3 N$ forces in $n-{ }^{3} \mathrm{He}$ and $p-{ }^{3} \mathrm{H}$ scattering. Work in this direction is in progress [70].
[1] S. Weinberg, Phys. Lett. B 251, 288 (1990); Nucl. Phys. B 363, 3 (1991); Phys. Lett. B 295, 114 (1992).

[2] C. Ordonez, L. Ray, and U. van Kolck, Phys. Rev. C 53, 2086 (1996).

[3] E. Epelbaum, H. W. Hammer, and U.-G. Meissner, Rev. Mod. Phys. 81, 1773 (2009).

[4] R. Machleidt and D. R. Entem, Phys. Rep. 503, 1 (2011).

[5] A. Deltuva, A. C. Fonseca, A. Kievsky, S. Rosati, P. U. Sauer, and M. Viviani, Phys. Rev. C 71, 064003 (2005).

[6] H. Witała, J. Golak, R. Skibiński, W. Glöckle, A. Nogga, E. Epelbaum, H. Kamada, A. Kievsky, and M. Viviani, Phys. Rev. C 73, 044004 (2006).
[7] H. Kamada, A. Nogga, W. Glöckle, E. Hiyama, M. Kamimura, K. Varga, Y. Suzuki, M. Viviani, A. Kievsky, S. Rosati, J. Carlson, S. C. Pieper, R. B. Wiringa, P. Navrátil, B. R. Barrett, N. Barnea, W. Leidemann, and G. Orlandini, Phys. Rev. C 64, 044001 (2001).

[8] B. S. Pudliner, V. R. Pandharipande, J. Carlson, S. C. Pieper, and R. B. Wiringa, Phys. Rev. C 56, 1720 (1997).

[9] R. B. Wiringa, S. C. Pieper, J. Carlson, and V. R. Pandharipande, Phys. Rev. C 62, 014001 (2000).

[10] A. Nogga, A. Kievsky, H. Kamada, W. Glöckle, L. E. Marcucci, S. Rosati, and M. Viviani, Phys. Rev. C 67, 034004 (2003). 
[11] R. Lazauskas and J. Carbonell, Phys. Rev. C 70, 044002 (2004).

[12] M. Viviani, A. Kievsky, and S. Rosati, Phys. Rev. C 71, 024006 (2005).

[13] P. Grassberger and W. Sandhas, Nucl. Phys. B 2, 181 (1967); E. O. Alt, P. Grassberger, and W. Sandhas, JINR report No. E4-6688 (1972).

[14] A. Deltuva and A. C. Fonseca, Phys. Rev. C 75, 014005 (2007).

[15] A. Deltuva and A. C. Fonseca, Phys. Rev. Lett. 98, 162502 (2007).

[16] A. Deltuva and A. C. Fonseca, Phys. Rev. C 76, 021001 (2007).

[17] E. O. Alt, W. Sandhas, and H. Ziegelmann, Phys. Rev. C 17, 1981 (1978); 21, 1733 (1980).

[18] A. Deltuva, A. C. Fonseca, and P. U. Sauer, Phys. Rev. C 71, 054005 (2005); 72, 054004 (2005).

[19] F. Ciesielski, Ph.D. Thesis, University J. Fourier, 1997 (unpublished); F. Ciesielski and J. Carbonell, Phys. Rev. C 58, 58 (1998); F. Ciesielski, J. Carbonell, and C. Gignoux, Phys. Lett. B 447, 199 (1999).

[20] R. Lazauskas, Ph.D. Thesis, University J. Fourier, 2003 (unpublished).

[21] R. Lazauskas and J. Carbonell, arXiv:nucl-th/0412068.

[22] R. Lazauskas, J. Carbonell, A. C. Fonseca, M. Viviani, A. Kievsky, and S. Rosati, Phys. Rev. C 71, 034004 (2005).

[23] R. Lazauskas, Phys. Rev. C 79, 054007 (2009).

[24] A. Kievsky, S. Rosati, M. Viviani, L. E. Marcucci, and L. Girlanda, J. Phys. G 35, 063101 (2008).

[25] M. Viviani, A. Kievsky, L. Girlanda, L. E. Marcucci, and S. Rosati, Few-Body Syst. 45, 119 (2009).

[26] M. Viviani, L. Girlanda, A. Kievsky, and L. E. Marcucci, Phys. Rev. Lett. 111, 172302 (2013).

[27] S. Aoyama, K. Arai, Y. Suzuki, P. Descouvemont, and D. Baye, Few-Body Syst. 52, 97 (2012).

[28] B. Pfitzinger, H. M. Hofmann, and G. M. Hale, Phys. Rev. C 64, 044003 (2001).

[29] H. M. Hofmann and G. M. Hale, Phys. Rev. C 68, 021002 (2003); 77, 044002 (2008).

[30] S. Quaglioni and P. Navrátil, Phys. Rev. Lett. 101, 092501 (2008); Phys. Rev. C 79, 044606 (2009).

[31] P. Navrátil, S. Quaglioni, G. Hupin, C. Romero-Redondo, and A. Calci, Phys. Scr. 91, 053002 (2016).

[32] M. Viviani, A. Deltuva, R. Lazauskas, J. Carbonell, A. C. Fonseca, A. Kievsky, L. E. Marcucci, and S. Rosati, Phys. Rev. C 84, 054010 (2011).

[33] A. Deltuva and A. C. Fonseca, Phys. Rev. Lett. 113, 102502 (2014); Phys. Rev. C 90, 044002 (2014).

[34] D. R. Entem and R. Machleidt, Phys. Rev. C 68, 041001 (2003).

[35] A. Hemmendinger, G. A. Jarvis, and R. F. Taschek, Phys. Rev. 76, 1137 (1949).

[36] R. S. Claassen, R. J. S. Brown, G. D. Freier, and W. R. Stratton, Phys. Rev. 82, 589 (1951).

[37] N. Jarmie and R. C. Allen, Phys. Rev. 114, 176 (1959).

[38] J. E. Brolley, Jr., T. M. Putnam, L. Rosen, and L. Stewart, Phys. Rev. 117, 1307 (1960).

[39] C. Manduchi, G. Moschini, G. Tornielli, and G. Zannoni, Nuovo Cimento B 57, 340 (1968).

[40] M. Ivanovich, P. G. Young, and G. G. Ohlsen, Nucl. Phys. A 110, 441 (1968).

[41] R. Kankowsky, J. C. Fritz, K. Kilian, A. Neufert, and D. Fick, Nucl. Phys. A 263, 29 (1976).
[42] J. D. Seagrave, L. Cranberg, and J. E. Simmons, Phys. Rev. 119, 1981 (1960).

[43] A. R. Sayres, K. W. Jones, and C. S. Wu, Phys. Rev. 122, 1853 (1961).

[44] V. P. Alfimenkov et al., Yad. Fiz. 33, 891 (1981).

[45] B. Haesner, W. Heeringa, H. O. Klages, H. Dobiasch, G. Schmalz, P. Schwarz, J. Wilczynski, B. Zeitnitz, and F. Käppeler, Phys. Rev. C 28, 995 (1983).

[46] C. E. Hollandsworth, M. Gilpatrick, and W. P. Bucher, Phys. Rev. C 5, 395 (1972).

[47] K. Sinram, F. W. Buesser, and F. Niebergall, Proc. of the Int. Conf. on Interact. of Neutr. with Nuclei (CONF-760715-P2), Lowell, edited by E. Sheldon et al. (University of Lowell Pub., 1976), p. 1363.

[48] H. O. Klages et al., Nucl. Phys. A 443, 237 (1985).

[49] P. Jany, W. Heeringa, H. O. Klages, B. Zeitnitz, and R. Garrett, Nucl. Phys. A 483, 269 (1988).

[50] J. Esterline, W. Tornow, A. Deltuva, and A. C. Fonseca, Phys. Rev. Lett. 110, 152503 (2013).

[51] J. H. Coon, Phys. Rev. 80, 488 (1950).

[52] R. Batchelor, R. Aves, and T. H. R. Skyrme, Rev. Sci. Instrum. 26, 1037 (1955).

[53] J. H. Gibbons and R. L. Macklin, Phys. Rev. 114, 571 (1954).

[54] J. Als-Nielsen and O. Dietrich, Phys. Rev. 133, B925 (1964).

[55] R. L. Macklin and J. H. Gibbons, Proc. of the Int. Conf. on the Study of Nuclear Structure with Neutrons, Antwerp, edited by M. Nève de Mévergnies, P. Assche, and J. Verbier (NorthHolland Pub., 1965), p. 13.

[56] D. G. Costello, S. J. Friesenhahn, and W. M. Lopez, Nucl. Sci. Eng. 39, 409 (1970).

[57] S. B. Borzakov, K. Maletski, L. B. Pikelner, M. Stempinski, and E. I. Sharapov, Yad. Fiz. 35, 532 (1982).

[58] G. A. Jarvis, A. Hemmendinger, H. V. Argo, and R. F. Taschek, Phys. Rev. 79, 929 (1950).

[59] H. B. Willard, J. K. Bair, and J. D. Kington, Phys. Rev. 90, 865 (1953).

[60] G. A. Jarvis, Los Alamos Scientific Report No. 2014, (1956), p. 35 .

[61] M. Drosg, Los Alamos Scientific Report No. 8215 (1980).

[62] D. S. Cramer and L. Cranberg, Nucl. Phys. A 171, 59 (1971).

[63] M. A. Doyle, Sr., H. W. Clark, L. J. Dries, J. L. Regner, T. R. Donoghue, and G. M. Hale, Nucl. Phys. A 371, 225 (1981).

[64] W. Tornow, R. C. Byrd, P. W. Lisowski, R. L. Walter, and T. R. Donoghue, Nucl. Phys. A 371, 235 (1981).

[65] J. R. Walston, C. D. Keith, C. R. Gould, D. G. Haase, B. W. Raichle, M. L. Seely, W. Tornow, W. S. Wilburn, G. W. Hoffmann, and S. I. Penttilä, Phys. Rev. C 58, 1314 (1998).

[66] W. S. Wilburn et al., Few-Body Syst. 24, 27 (1998).

[67] M. Abramowitz and I. Stegun, Handbook of Mathematical Functions (Dover Publications, Inc., New York, 1970).

[68] M. Viviani, Few-Body Syst. 25, 177 (1998).

[69] B. M. Fisher, C. R. Brune, H. J. Karwowski, D. S. Leonard, E. J. Ludwig, T. C. Black, M. Viviani, A. Kievsky, and S. Rosati, Phys. Rev. C 74, 034001 (2006).

[70] M. Viviani, L. Girlanda, A. Kievsky, and L. E. Marcucci (unpublished).

[71] L. D. Faddeev, Zh. Eksp. Teor. Fiz. 39, 1459 (1960) [Sov. Phys. JETP 12, 1014 (1961)].

[72] O. A. Yakubovsky, Sov. J. Nucl. Phys. 5, 937 (1967).

[73] D. Baye, Phys. Rep. 565, 1 (2015). 